\title{
ANALGESIC UTILIZATION IN POSTOPERATIVE CARE AT A TERTIARY CARE TEACHING HOSPITAL IN NEPAL
}

\author{
Rauniar G P*, Naga Rani M A*, Das B P*, Agrawal C S
}

\section{ABSTRACT}

A retrospective analysis of analgesics used in postoperative pain in abdominal surgery was done to develop base line data. Appendectomy and cholecystectomy each formed $38.63 \%$ while hernia repair constituted $\mathbf{2 2 . 7 4 \%}$ of all the abdominal surgeries (422). NSAIDs were the most commonly prescribed group of analgesics both during the hospital stay $(99.52 \%)$ and at the time of discharge $(86.01 \%)$. Opioid analgesics $(4.73 \%)$ was used in the immediate postoperative period mainly to supplement the NSAIDs. Diclofenac $(\mathbf{8 8 . 8 6 \% )}$ was the most commonly prescribed analgesic to in-patients and either diclofenac (47.63) or a fixed dose combination of ibuprofen and paracetamol $(32.46 \%)$ was the most commonly prescribed at the time of discharge. The administered dose of diclofenac was higher than the recommended dose. Utilization of analgesics during postoperative period may be rationalised by pain assessment charts and regular feedback to the precribers.

\section{Key Words: Analgesic utilization, post operative, abdominal surgery.}

\section{INTRODUCTION}

Pain control is one of the common problems encountered in medical treatment. Optimal relief from postoperative pain will facilitate early mobility and recovery reducing the length of the stay in the hospital. Measures for alleviation of postoperative pain in general ${ }^{1}$ and in selected procedures such as abdominal surgery ${ }^{2}$ have been clearly outlined. However, a multi-center study in Spain involving 12 hospitals revealed a wide inter-hospital variation in the management of postoperative pain. It was found that due to under-utilization of analgesics, 22 to $67 \%$ of patients suffered from unbearable pain in the postoperative period. One of the reasons for underutilization of analgesics in hospital practice may be because of not quantifying pain routinely. Optimal pain control is an important component of good patient care and needs to be encouraged by feedback to the clinicians on prescribing practice and pain relief score. There is no data available on analgesic utilization in postoperative pain in Nepal. Hence, the present retrospective study was undertaken to develop baseline data on the pattern of prescribing of analgesics in postoperative pain at B. P. Koirala Institute of Health Sciences, Dharan, Nepal and provide feedback to the clinicians to optimize their prescribing practice.

\section{METHODS}

The case records of all patients who had undergone abdominal surgery between April 1998 and March 1999 (1 year) were analysed using a standard proforma to collect data on demography of patients, type of surgical procedure (according to the duration and complexity) and analgesic prescription in

* B.P. Koirala Institute of Health Sciences, Dharan, Nepal.

Address for correspondence : $\quad$ Dr. G. P. Rauniar

Department of Pharmacology

B.P. Koirala Institute of Health Sciences, Dharan, Nepal.

Email: gprauniar@hotmail.com 
major (appendectomy \& cholecystectomy) and minor surgical procedures (inguinal hernia repair). ${ }^{3}$

\section{RESULTS}

A total number of 422 patients underwent abdominal surgery and their mean (SD) age was 32.59 (SD 12.00). Table I provides the demographic data of the patients. Table II \& III provide data on the prescribing pattern of analgesics group-wise and drug-wise. The data revealed that NSAIDs were the most commonly prescribed group of analgesics both during hospital stay $(99.52 \%)$ and at the time of discharge (86.01\%). Opioids were prescribed in 18 patients in the immediate postoperative period to supplement NSAIDs when NSAIDs alone could not control the pain. Since our data was taken from the nursing remarks, it indicates exact usage. But, as it was a retrospective study, the drawback was inability to know the exact degree of pain relief. Analgesics were most commonly prescribed by intramuscular administration through a fixed schedule in the immediate postoperative period and by oral administration in 